\title{
Wave propagation in single- and double-walled carbon nanotubes filled with fluids
}

\begin{abstract}
Wave propagation approach of single- and double-walled carbon nanotubes conveying fluid is presented through the use of the continuum mechanics. A simplified Flügge shell equations are proposed as the governing equations of motion for carbon nanotubes studied here. For the double-walled nanotubes, the deflection of nested tubes is considered to be coupled through the van der Waals interaction between two adjacent nanotubes. Effects of filled fluid property and nanotube diameter on the wave propagation are investigated and analyzed based on the proposed elastic continuum model. The theoretical investigation may give a useful reference for potential design and application of nanoelectronics and nanodevices.
\end{abstract}

Key words:

62.30. $+\mathrm{d} \quad$ Mechanical and elastic waves; vibrations

62.25. $\mathrm{g}$ Mechanical properties of nanoscale materials

62.40.+I Anelasticity, internal friction, stress relaxation, and mechanical resonances

Toshiaki Natsuki* and Qing- Qing Ni

Faculty of Textile Sicence \& Technology, Shinshu University,

3-15-1 Tokida, Ueda-shi, 386-8567, Japan

Morinobu Endo

Faculty of Engineering, Shinshu University,

Wakasato 4-17-1, Nagano-shi, 380-8553, Japan

* Corresponding author

E-mail: natsuki@shinshu-u.ac.jp

TEL: 81-0268-21-5421, FAX: 81-0268-21-5482 


\section{INTRODUCTION}

Carbon nanotubes (CNTs) have been shown to possess remarkable mechanical [1-3] and physical properties leading to many potential applications [4-8]. In particular, CNTs hold substantial promise as superfibers for composite materials $[2,4,5]$. Hence, understanding the mechanical and physical properties of CNTs is essential for their applications. The study of vibration and wave propagation in CNTs is a major topic of current interest, which is used to understand the dynamic behaviors of CNTs. Many experimental [1-3,9] and theoretical methods [10-15] have been presented for measuring and predicting the stiffness and strength properties of CNTs. Some studies on the vibration [16-18] and the wave propagation [19-21] have been done. Since CNTs are extremely small, the experiments to measure the properties of individual CNT are quitedifficult . Therefore, the computational simulations have been regarded as a powerful tool to study properties of CNTs. There are two major categories for simulating the mechanical properties of CNTs: molecular dynamics (MD) simulation and continuum mechanics. Although the MD method has been successfully used for simulating the mechanical and physical properties of structures at atomic-scale level, this method is time-consuming and remains formidable especially for larger-scale systems. Recently, solid mechanics with elastic continuum model have been regarded as an effective method and widely used for studing the mechanical and physical properties of CNTs [16,18-24].

Yoon et al. have studied the transverse vibration and wave propagation in multi-walled 
carbon nanotubes (MWNTs) using Bernouli-Euler beam model $[16,19,20]$. The analytical solutions were limited to the one-dimensional model since CNTs are modeled as beams. Recently, the vibration of MWNTs and wave propagation of double walled carbon nanotubes (DWNTs) have been studied based on Flügge shell equation [18,21]. The results showed that the CNTs have sound wave frequencies over terahertz because of their nanoscale, which opens a new topic on wave characteristics. Wang and Varadan [24] have presented the elastic wave solution obtained from Euler-Bernoulli beam and Timoshenko beam models. They reported that the comparison between the two models is inappropriate on the terahertz frequency range. This suggested that the Timoshenko beam model should be employed in analyzing the wave propagation for the high frequency range.

Because of the superior mechanical behaviors and hollow geometry, CNTs hold substantial promise as nanopipes for conveying fluid or gas. The study of CNTs filled with fuilds is a challenging topic of current interest [25-27]. Therefore, it is very significant to study the vibration and the wave propagation of CNTs conveying fluid. The present work presents a theoretical approach to investigate the wave propagation of single- and double-walled CNTs conveying fluids. The constitutive behavior of CNTs is described by Flügge shell equation, and the motion equation of fluids is given by Morse and Ingard [28]. Using the proposed method, the influence of fluid properties on the wave propagation in CNTs conveying fluid is investigated and analyzed. 


\section{II . THEORETICAL APPROACH}

\section{A. Fundamental equations}

Approximate Flügge shell equations are proposed as the governing equations of vibration for CNTs. Figure 1 shows a cylindrical coordinate system of the CNT shell model. The $x$ coordinate is taken in the axial direction of the shell, where the $\theta$ and $z$ coordinates are in the circumferential and radial directions, respectively. The displacements of the nanotube are defined by $u, v$ and $w$ in the direction of $x, \theta$ and $z$-axes, respectively. The CNT has a thickness of $h$, radius of $R$ and length of $L$.

The equations of motion for nanotubes can be obtained based [21] on Love's first approximation shell theory [28]

$$
\left[\begin{array}{ccc}
L_{1} & L_{2} & L_{3} \\
L_{2} & L_{4} & L_{5} \\
-L_{3} & -L_{5} & L_{6}
\end{array}\right]\left\{\begin{array}{l}
u \\
v \\
w
\end{array}\right\}=\left\{\begin{array}{c}
0 \\
0 \\
-p K
\end{array}\right\}
$$

where $L_{j}(j=1, \Lambda, 6)$ are the differential operators with respect to $x$ and $\theta$ given by

$$
\begin{aligned}
& L_{1}=\frac{\partial^{2}}{\partial x^{2}}+\frac{1-\sigma}{2 R^{2}} \frac{\partial^{2}}{\partial \theta^{2}}-K \rho h \frac{\partial^{2}}{\partial t^{2}}, \quad L_{2}=\frac{1+\sigma}{2 R} \frac{\partial^{2}}{\partial x \partial \theta}, \quad L_{3}=-\frac{\sigma}{R} \frac{\partial}{\partial x}, \\
& L_{4}=\frac{1-\sigma}{2} \frac{\partial^{2}}{\partial x^{2}}+\frac{1}{R^{2}} \frac{\partial^{2}}{\partial \theta^{2}}+\alpha\left[(1-\sigma) \frac{\partial^{2}}{\partial x^{2}}+\frac{1}{R^{2}} \frac{\partial^{2}}{\partial \theta^{2}}\right]-K \rho h \frac{\partial^{2}}{\partial t^{2}}, \\
& L_{5}=\frac{1}{R^{2}} \frac{\partial}{\partial \theta}-\alpha\left[(2-\sigma) \frac{\partial^{3}}{\partial x^{2} \partial \theta}+\frac{1}{R^{2}} \frac{\partial^{3}}{\partial \theta^{3}}\right], \\
& L_{6}=-\frac{1}{R^{2}}-\alpha\left(R^{2} \frac{\partial^{4}}{\partial x^{4}}+2 \frac{\partial^{4}}{\partial x^{2} \partial \theta^{2}}+\frac{1}{R^{2}} \frac{\partial^{4}}{\partial \theta^{4}}\right)-K \rho h \frac{\partial^{2}}{\partial t^{2}},
\end{aligned}
$$


where $K=\left(1-v^{2}\right) / E h, \alpha=h^{2} / 12 R^{2} . E$ and $v$ are the elastic modulus and Poisson's ratio of agraphe ne sheet folded into CNTs.

Eliminating $u$ and $v$ from Eq. (1), we have

$$
L_{A} w+L_{B}(p K)=0
$$

where

$$
\begin{aligned}
& L_{A}=L_{5}\left(L_{2} L_{3}-L_{1} L_{5}\right)+L_{3}\left(L_{2} L_{5}-L_{3} L_{4}\right)+L_{6}\left(L_{2} L_{2}-L_{1} L_{4}\right), \\
& L_{B}=\left(L_{2} L_{2}-L_{1} L_{4}\right) .
\end{aligned}
$$

The motion equation of fluid in the cylindrical shell can be given by [29]

$$
\frac{1}{r} \frac{\partial}{\partial r}\left(r \frac{\partial p}{\partial r}\right)+\frac{1}{r^{2}} \frac{\partial^{2} p}{\partial \theta^{2}}+\frac{\partial^{2} p}{\partial x^{2}}=\frac{1}{c_{f}^{2}} \frac{\partial^{2} p}{\partial t^{2}}
$$

where $p$ is the acoustic pressure and $c_{f}$ is the sound speed of the fluid.

\section{B. Wave propagation approach}

In the coordinate system as shown in Fig. 1, the displacement of nanotubes in the $\mathrm{z}$ direction can be expressed in the form of wave propagation, associated with the axial wave number $k_{m}$ and the angular wave number $n$. DWNTs are assumed to be two individual coaxial tubes coupled together through van der Waals (vdW) interaction between the inner and outer nanotubes. The general solution form of the wave propagation can be given by

$$
w_{j}=A_{j} \exp \left[i\left(k_{m} x+n \theta-\omega t\right)\right]
$$

where $A_{j}(j=1,2)$ are the amplitude of vibration in the inner and outer nanotubes, and $\omega$ is 
the circular frequency.

The solution form of the acoustic pressure field of the cylindrical shell filled with fluid, which satisfies Eq. (5), can be expressed as

$$
p_{f}=P_{0} Z_{n}\left(k_{r} r\right) \exp \left[i\left(k_{m} x+n \theta-\omega t\right)\right]
$$

where $Z_{n}$ denotes a Bessel function $J_{n}$ of order $n$ when $k_{r}^{2}=\omega^{2} / c_{f}^{2}-k_{m}^{2}>0$, and the modified Bessel function $I_{n}$ when $k_{r}^{2}=k_{m}^{2}-\omega^{2} / c_{f}^{2}>0 . k_{r}$ and $k_{m}$ present the radial and axial wave numbers, respectively.

Since the fluid remains in contact with the tube wall, the fluid displacement is equal to the radial displacement of the inner tube wall. This coupling condition is given by Fuller and Fahy [30] from the momentum equation. Thus, the fluid pressure amplitude to be written in terms of the radial displacement amplitude as

$$
P_{0}=\frac{\rho_{f} \omega^{2} A_{1}}{k_{r} Z_{n}^{\prime}\left(k_{r} r_{1}\right)}
$$

where $\rho_{f}$ is the density of the fluid. $A_{1}$ is the amplitude of vibration in the inner nanotube.

Substituting Eq. (8) into Eq. (7), and using Eq. (6), we obtain

$$
p_{f}=k_{f}\left(\omega, k_{r}\right) w_{1}, \quad k_{f}\left(\omega, k_{r}\right)=\frac{\rho_{f} \omega^{2} Z_{n}\left(k_{r} r\right)}{k_{r} Z_{n}^{\prime}\left(k_{r} r\right)} .
$$

DWNTs are regarded as nested SWNTs whose pressure between two adjacent nanotubes results from the vdW interaction. The vdW interaction energy potential, as a function of the interlayer spacing between the inner and outer nanotubes, can be estimated by the 
Lennard-Jones potential. Since the interlayer spacing of DWNTs is very close to equilibrium spacing, the initial van vdW force remains zero for each of the tubes provided they deform coaxially. In case of small-amplitude vibration of CNTs, the interaction pressure between two adjacent nanotubes is assumed to be linear relative to the difference of their deflections in the radial direction. Therefore, the coupled equations of the pressure $(p)$ in the Eq. (4) caused by the vdW interaction in DWNTs can be given by

$$
\begin{aligned}
& p_{1}=p_{f}+c_{21}\left(w_{2}-w_{1}\right) \\
& p_{2}=c_{12}\left(w_{1}-w_{2}\right)
\end{aligned}
$$

where $w_{1}$ and $w_{2}$ are the deflections in the inner and outer nanotubes, respectively.

In this simulation, the vdW interaction coefficient $c_{i j}$ can be estimated as [31]

$$
c_{i j}=\frac{\pi \varepsilon R_{j} \sigma^{6}}{a^{4}}\left[\frac{1001 \sigma^{6}}{3} H_{i j}^{13}-\frac{1120}{9} H_{i j}^{7}\right],
$$

where

$$
H_{i j}^{m}=\left(R_{i}+R_{j}\right)^{-m} \int_{0}^{\pi / 2} \frac{d \theta}{\left(1-K_{i j} \cos ^{2} \theta\right)^{m / 2}}, \quad(m=7,13),
$$

and

$$
K_{i j}=\frac{4 R_{i} R_{j}}{\left(R_{i}+R_{j}\right)^{2}}
$$

where $\sigma$ and $\varepsilon$ are the depth of the Lennard-Jones potential and vdW radius, respectively.

Substituting Eq. (10) into Eq. (3), the coupled equations of the wave speed in DWNTs are written as 


$$
\begin{aligned}
& L_{A}^{1} w_{1}+L_{B}^{1}\left[K k_{f}\left(\omega, k_{r}\right) w_{1}+c_{21} K\left(w_{2}-w_{1}\right)\right]=0, \\
& L_{A}^{2} w_{2}+L_{B}^{2}\left[c_{12} K\left(w_{1}-w_{2}\right)\right]=0
\end{aligned}
$$

By substituting Eqs. (7) and (9) into Eq. (14), the phase velocity in DWNTs can be determined by a nontrivial solution in Eq. (14) as

$$
\left|\begin{array}{cc}
L_{A}^{1}\left(\omega, k_{x}, n\right)+K\left[k_{f}\left(\omega, k_{r}\right)-c_{21}\right] L_{B}^{1}\left(\omega, k_{x}, n\right) & c_{21} K L_{B}^{1}\left(\omega, k_{x}, n\right) \\
c_{12} K L_{B}^{2}\left(\omega, k_{x}, n\right) & L_{A}^{2}\left(\omega, k_{x}, n\right)-c_{12} K L_{B}^{2}\left(\omega, k_{x}, n\right)
\end{array}\right|=0 .
$$

where $L_{A}^{j}$ and $L_{B}^{j}(j=1,2)$ are the differential operators, which are given by Eqs. (2) and (4) for inner and outer tubes, respectively.

The amplitude ratio of phase velocity is given by

$$
\frac{A_{1}}{A_{2}}=\frac{-c_{21} K L_{B}^{1}\left(\omega, k_{x}, n\right)}{L_{A}^{1}\left(\omega, k_{x}, n\right)+K\left[k\left(\omega, k_{r}\right)-c_{21}\right] L_{B}^{1}\left(\omega, k_{x}, n\right)}
$$

For SWNTs, the solution of the wave velocity is given by a polynomial function of $L_{A}(\omega, k, n)=0$. The frequency dependence of the phase velocity $\left(c_{p}\right)$ can be obtained in terms of $c_{p}=\omega / k$

\section{NUMERICAL RESULTS AND DISCUSSION}

Influences of fluid properties on the natural frequency of CNTs were investigated using the proposed method. In this simulation, the thickness of individual SWNT was assumed to be that of a graphite sheet with $0.34 \mathrm{~nm}$. CNTs had an elastic modulus of $1 \mathrm{TPa}$, Poisson's ratio of 0.27 , and the density of $2.0 \mathrm{~g} / \mathrm{cm}^{3}$. The vdW parameters used in the Lennard-Jones potential are taken as $\varepsilon=2.967 \mathrm{meV}$ and $\sigma=0.34 \mathrm{~nm}$ reported recently by Saito et al. [32] 
For following solution of the natural frequency in CNTs, we will discuss the low-frequency mode of $m=n=1$.

Figures 2 shows the dispersion curves of wave speed with different frequencies for SWNTs filled with the fluids of different properties. The SWNTs have a diameter of $3.0 \mathrm{~nm}$. The property of water fluid has the density of $1000 \mathrm{~kg} / \mathrm{m}^{3}$ and the free wave speed of 1530 $\mathrm{m} / \mathrm{s}$. It is found that wave speeds rapidly increases with increasing frequency and reaches a peak. The peaks of the wave speed and its frequency are affected by the properties of filled fluids, and the peak values are approximately $2 \mathrm{~km} / \mathrm{s}$ with a frequency of about $1.5 \mathrm{THz}$. After this peak, the analytical wave speed decreases with increasing frequency and then remains fairly to constants. It is also noticed that there exist several terahertz critical frequencies in CNTs. The critical frequency is commonly called "cut-off frequency," given by when the frequency the critical frequency. In physics, the cut-off frequency of an electromagnetic wave is the lowest frequency for which a mode will propagate in it. The critical frequencies are largely affected by the wave speed of filled fluids. For the CNT filled with water fluid shown in Fig. 2(b), the lowest critical frequency is about $5.5 \mathrm{~Hz}$. The second and the third critical frequencies are $8.7 \mathrm{THz}$ and $12.0 \mathrm{THz}$, respectively. Figure 3 shows the dispersion curves of wave speed for a CNT with $2.0 \mathrm{~nm}$ diameter and filled with water fluid. The lowest and the second critical frequencies are about $8.2 \mathrm{~Hz}$ and $13.4 \mathrm{~Hz}$, respectively. Compared with the curve of the wave velocity shown in Fig. 2(b), the critical frequencies in CNTs will increase 
with a decrease in the diameter of the CNTs.

Figure 4 shows dispersion curves of wave propagation for DWNTs filled with water. The result is quite different from those obtained from the SWNT. As the frequency is increased, the wave speed of DWNTs rises rapidly and has the lowest critical frequency of $1.2 \mathrm{THz}$. The other several critical frequencies that are similar to those in the SWNTs still exist in the DWNTs. The amplitude ratio of wave speeds in the inner to outer layers is shown in Fig. 5 as a function of the vibration frequency. It is seen that the amplitude ratio $\left(A_{1} / A_{2}\right)$ is close to 1 when the frequency is less than $0.7 \mathrm{THz}$. This indicates that the vibration mode is the coaxial for lower frequency and that the deflection of the inner to outer tubes has the same amplitude. When the wave speed is close to the critical frequency of $1.2 \mathrm{THz}$, the amplitude ratio of the inner to outer tube deflections rapidly increases. For the vibration frequency over $2.5 \mathrm{THz}$, the inner tube deflection within DWNTs is larger than the outer tube deflection.

\section{CONCLUSIONS}

A theoretical approach based on a continuum elastic shell model is proposed to study the sound wave propagation in both single- and double-walled CNTs filled with fluids. Using the proposed theoretical approach, the effects of nanotube diameter and fluid properties on the wave propagation are investigated. It is found that there exist several critical frequencies in

CNTs. The wave propagation in fluid-filled CNTs is affected largely by the free wave speed 
of the fluids. When a vibration frequency is lower than the lowest critical frequency (less than $0.5 \mathrm{THz}$ ), the vibration mode is the coaxial and the wave deflection of the inner to outer tubes has the same amplitude.

\section{ACKNOWLEDGEMEN}

This work was supported by the CLUSTER of Ministry of Education, Culture, Sports, Science and Technology (Japan). 


\section{REFERENCES}

${ }^{1}$ E. W. Wong, P. E. Sheehan and C. M. Lieber, Scince 277, 1971 (1997).

${ }^{2}$ M. M. J. Treacy, T. W. Ebbesen and J. M. Gibson, Nature (London) 381, 678 (1996).

3 B. I. Yakobson and Ph. Avouris, Carbon nanotubes, edited by M.S. Dresselhaus (Springer Verlag, Berlin-Heidelberg, 2001) chap 9, p.287.

4 K. T. Lau and D. Hui, Composites, Part B 33, 263 (2002).

${ }^{5}$ D. Qian, G. J. Wagner, W. K. Liu, M. -F. Yu and R. S. Appl. Mech. Rev. 55, 495 (2002).

${ }^{6}$ E. T. Thostenson, Z. Ren and T. W. Chou, Comp. Sci. Technol. 61, 1899 (2001).

${ }^{7}$ V. N. Popov, Mater. Sci. Eng. R43, 61 (2004).

${ }^{8}$ V. Derycke, R. Martel, J. Appenzeller and Ph. Avouris, Nano Lett. 1, 453 (2001).

${ }^{9}$ M. F. Yu, O. Lourie, M. J. Dyer, K. Moloni, T. F. Kelly and R. S. Ruoff, Science 287, 637 (2000).

${ }^{10}$ B. I. Yakobson, C. J. Brabec and J. Bernholc, Phys. Rev. Lett. 76, 2511 (1996).

${ }^{11}$ B. I. Yakobson, Appl. Phys. Lett. 72, 918 (1998).

${ }^{12}$ K. N. Kudin, G. E. Scuseria and B. I. Yakobson, Phys. Rev. B 64, 235406 (2001).

${ }^{13}$ P. Zhang, P. E. Lammert and V. H. Crespi, Phys. Rev. Lett. 81, 5346 (1998).

${ }^{14}$ T. Natsuki and M. Endo, Appl. Phys. A 80, 1463 (2005).

${ }^{15}$ P. Zhang, P. E. Lammert and V. H. Crespi, Phys. Rev. Lett. 81, 5346 (1998)

${ }^{16}$ J. Yoon, C. Q. Ru and A. Mioduchowski, Compos. Sci. Technol. 63, 1533 (2003). 
${ }^{17}$ P. C. Eklund, J. M. Holden and R. A. Jishi, Carbon 33, 959 (1995).

${ }^{18}$ C. Y. Wang, C. Q. Ru and A. Mioduchowski, Phys. Rev. B 72, 075414 (2005).

${ }^{19}$ J. Yoon, C. Q. Ru and A. Mioduchowski, J. Appl. Phys. 93. 4801 (2003).

${ }^{20}$ J. Yoon, C. Q. Ru and A. Mioduchowski, Composites B 35, 87 (2004).

21 T. Natsuki, T. Hayashi and M. Endo, J. Appl. Phys. 97044307 (2005)

22 C. Q. Ru, Phys. Rev. B 62, 16962 (2000).

23 X. Q. He, S. Kitipornchai, K. M. Liew, J. Mech. Phys. Solids 53. 303 (2005).

24 Q. Wang and V. K. Varadan, Int. J. Solids Struc. 43. 254 (2006).

25 G. Hummer, J. C. Rasaiah and J. P. Noworyta, Nature 414, 188 (2001).

26 Y. Gao and Y. Bando, Nature 415, 599 (2002).

27 C. M. Megaridis, A. G. Yazicioglu, J. A. Libera, Y. Gogotsi, Physics of Fluids 14. L5 (2002).

28 A. W. Leissa, Vibration of Shells (Scientific and Technical Information Office, National Aeronautics and Space Administration, Washington, 1973)

29 P. M. Morse and K. U. Ingard, Theoretical acoustics. (New York: McGraw-Hill Book Company; 1968)

30 C. R. Fuller and F. J. Fahy, J. Sound Vib. 81, 501 (1982).

31 X.Q. He S. Kitipornchai, K.M. Liew, J. Mech. Phys. Solids 53, 303 (2005).

32 R. Saito, R Matsuo, T. Kimura, G. Dresselhaus and M. S. Dresselhaus, Chem. Phys. Lett. 
338, 187 (2001). 


\section{Figure captions}

Figure 1 Geometry of nanotube and coordinate system employed in the analysis

Figure 2 Dispersion curves of wave propagation in SWNTs with a diameter of $3.0 \mathrm{~nm}$. The properties of fluids are (a) density $\rho_{f}=500 \mathrm{~kg} / \mathrm{m}^{3}$, sound speed $c_{f}=1530 \mathrm{~m} / \mathrm{s}$;

$$
\begin{aligned}
& \text { (b) density } \rho_{f}=1000 \mathrm{~kg} / \mathrm{m}^{3} \text {, sound speed } c_{f}=1530 \mathrm{~m} / \mathrm{s} \text {; } \\
& \text { (c) density } \rho_{f}=1000 \mathrm{~kg} / \mathrm{m}^{3} \text {, sound speed } c_{f}=1800 \mathrm{~m} / \mathrm{s}
\end{aligned}
$$

Figure 3 Dispersion curves of wave propagation in SWNTs with a diameter of $2.0 \mathrm{~nm}$. The properties of fluid: density $\rho_{f}=1000 \mathrm{~kg} / \mathrm{m}^{3}$, sound speed $c_{f}=1530 \mathrm{~m} / \mathrm{s}$

Figure 4 Dispersion curves of wave propagation in DWNTs with the inner and outer diameters of $2.2 \mathrm{~nm}$ and 3.0, respectively. The properties of fluid: density $\rho_{f}=1000 \mathrm{~kg} / \mathrm{m}^{3}$, sound speed $c_{f}=1530 \mathrm{~m} / \mathrm{s}$.

Figure 5 Amplitude ratio of the inner to the outer tube deflections in DWNTs with the inner and outer diameters of $2.2 \mathrm{~nm}$ and 3.0, respectively. The properties of fluid: density $\rho_{f}=1000 \mathrm{~kg} / \mathrm{m}^{3}$, sound speed $c_{f}=1530 \mathrm{~m} / \mathrm{s}$. 
Figure $1 \quad$ (T. Natsuki)

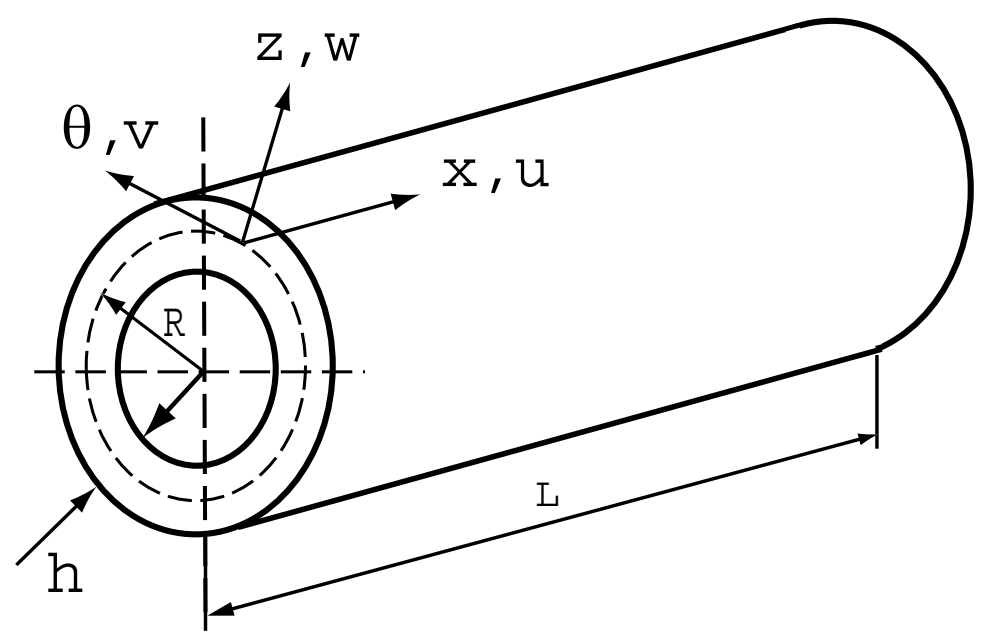


Figure 2 (T. Natsuki)
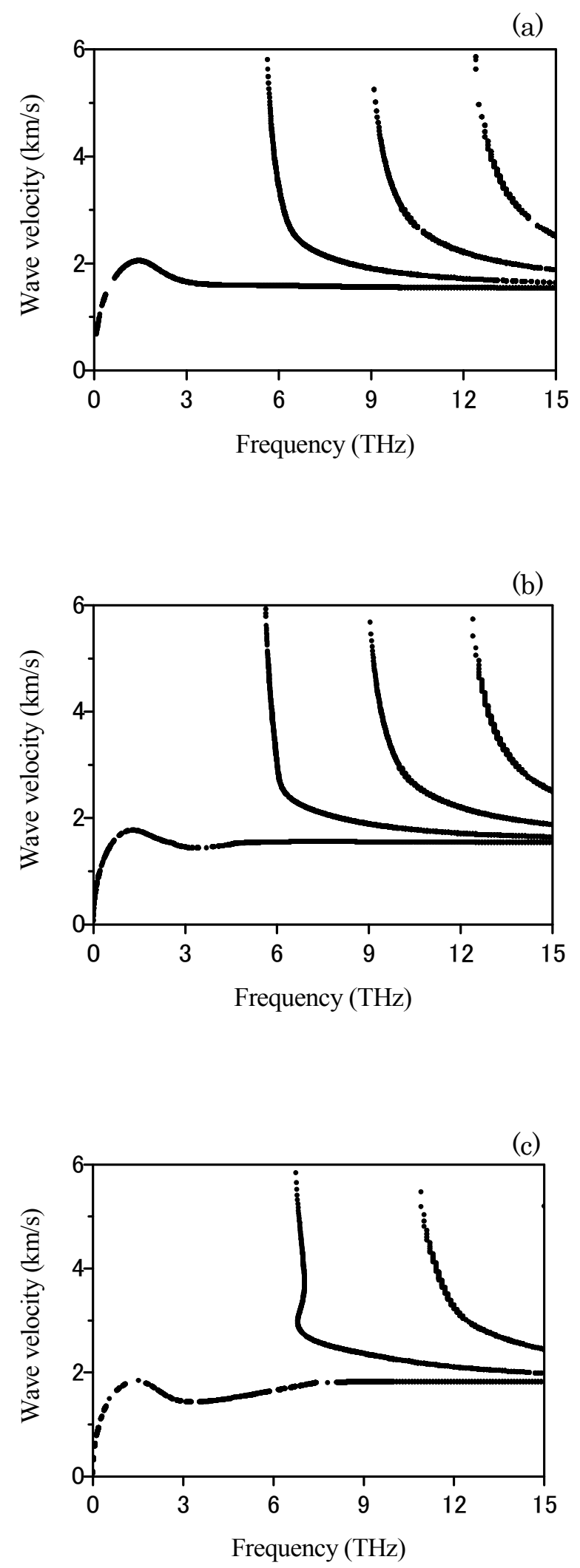
Figure 3 (T. Natsuki)

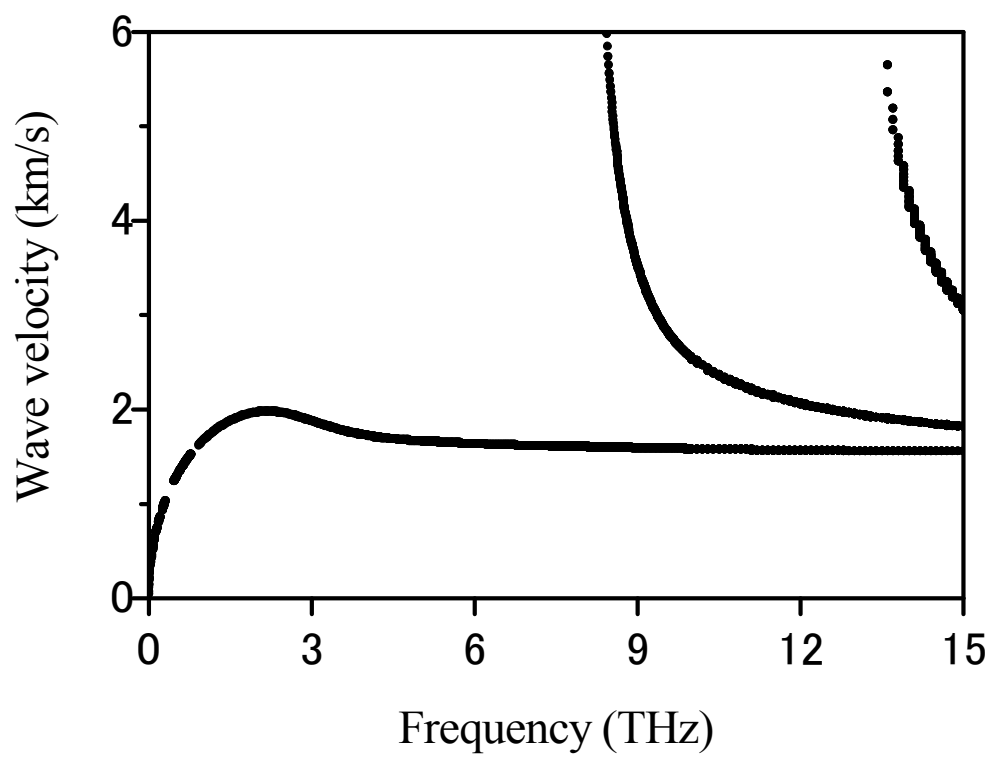


Figure $4 \quad$ (T. Natsuki)

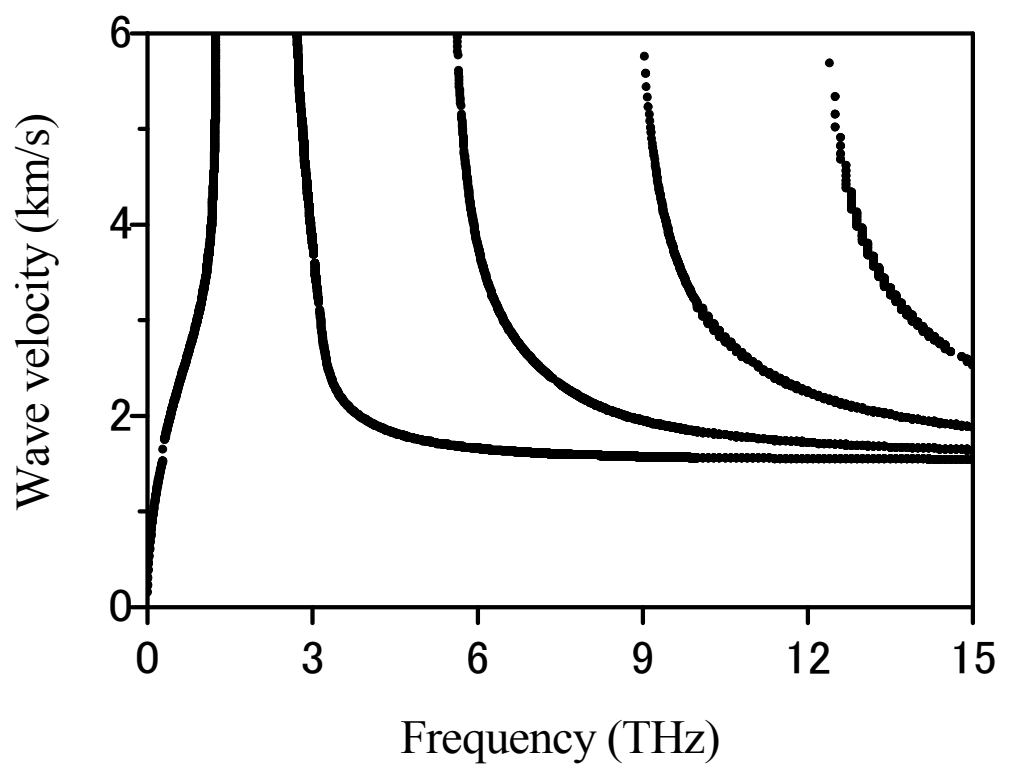


Figure $5 \quad$ (T. Natsuki)

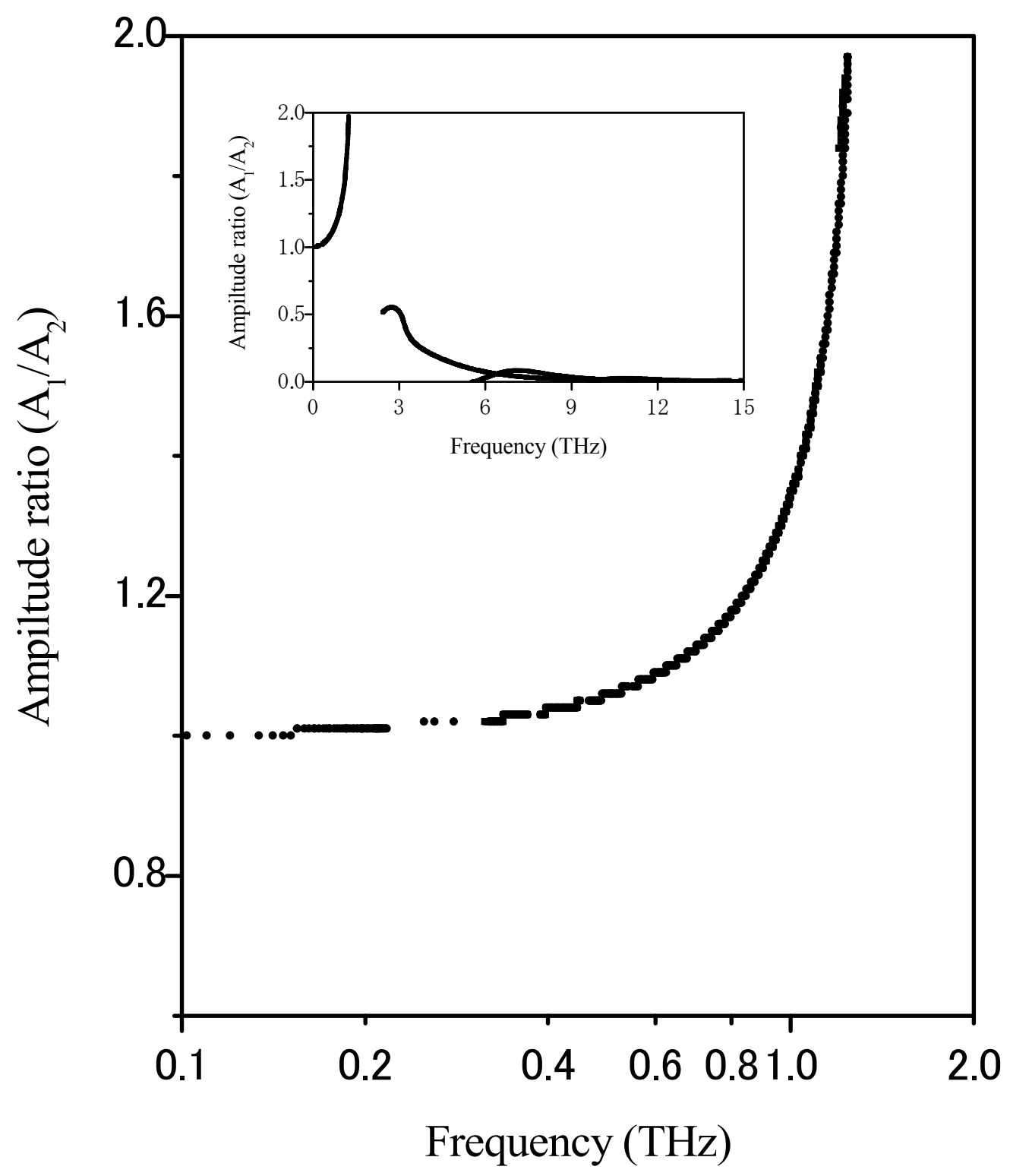

